\title{
Searching for cognitive processes underlying insect learning
}

\author{
MAKOTO MizUnAMI $^{1)}$, ChIHIRO SATO-MATSUMOTO ${ }^{2)}$ \\ and YUKIHISA MATSUMOTO ${ }^{21}$
}

\begin{abstract}
Elucidation of neural mechanisms of learning and memory in insects and their comparison with those in mammals should help to deepen our understanding of evolution of the brain and behavior in animals. Our studies on Pavlovian (classical) conditioning in crickets suggested that octopamine (OA), the invertebrate counterpart of noradrenaline, and dopamine (DA) convey signals of appetitive and aversive unconditioned stimulus (US), respectively. Our studies also suggested that activation of OA or DA neurons is needed for execution of appetitive or aversive conditioned response (or for appetitive or aversive memory retrieval), respectively. We proposed that Pavlovian conditioning in crickets is determined by prediction error, i.e., discrepancy between the actual US and predicted US, as has been suggested in mammals. OA neurons appear to mediate the prediction error signals in appetitive conditioning. We conclude that insect Pavlovian conditioning is based on sophisticated information processing that shares many features with those in mammals, suggesting evolutionary convergence of basic brain functions between mammals and insects.
\end{abstract}

Key words : Pavlovian conditioning, octopamine, dopamine, prediction error, insects

\section{Introduction}

Insects exhibit many sophisticated behaviors, all of which are controlled by their small brains, which we refer to as "microbrains" (Mizunami, Yokohari, \& Takahata, 1999, 2004). Elucidation of the functional organizations of insect microbrains and comparison of them with mammalian "megalobrains" should help to elucidate common principles of animal brains across phyla, as well as specific adaptations in each group. We investigated neural mechanisms of Pavlovian (classical) conditioning, a basic form of associative learning, in insects and

1) Faculty of Science, Hokkaido University Kita 10 Nishi 8, Kita-ku, Sapporo, 060-0810

2) College of Liberal Arts and Science, Tokyo Medical and Dental University

2-80-30, Kokubudai, Ichikawa, Chiba, 272-0827

Corresponding author: MAKOTO MizUNAMI

E-mail: mizunami@sci.hokudai.ac.jp

J-STAGE Advance Published Date: June 22, 2017. doi: 10.2502/janip.67.1.5 compared them with those in mammals. We focused on the roles of octopamine (OA) and dopamine (DA) in conveying appetitive and aversive signals, respectively, in the cricket Gryllus bimaculatus.

In this article we first briefly describe procedures to study learning and memory in crickets and then summarize our studies using pharmacology, RNAi and transgenesis on the roles of $\mathrm{OA}$ and DA neurons in mediating appetitive and aversive signals in classical conditioning. The results are compared with findings in mammals, and conservation of basic computational principles underlying associative learning among insects and mammals is discussed.

Crickets have several advantages for the study of neural mechanisms of learning and memory (Mizunami \& Matsumoto, 2010; Mizunami, Matsumoto, Watanabe, \& Nishino, 2013). At first, they have excellent capabilities for olfactory and visual learning. Secondly, knowledge has been accumulated on the brain and behavior since they have been 
used as materials for the study of neural basis of behavior. Thirdly, pharmacological approaches (Unoki, Matsumoto, \& Mizunami, 2005, 2006; Matsumoto, Unoki, Aonuma, \& Mizunami 2006,; Matsumoto, Hatano, Unoki \& Mizunami, 2009; Matsumoto, Hirashima, Terao, \& Mizunami, 2013), gene knockdown by RNA interference (Takahashi, Matsumoto, \& Mizunami, 2009; Awata, Wakuda, Ishimaru, Matsuoka, Terao, Katata, Matsumoto, Hamanaka, Noji, Mito, \& Mizunami, 2016), and genome editing by CRISPR/cas9 system (Awata, Watanabe, Hamanaka, Mito, Noji, \& Mizunami, 2015) have been well established in crickets, which allows detailed analysis of molecular basis of learning and memory. Finally, as we will discuss in this article, conditioning with different conditioned stimuli (CSs) (odor, visual pattern and color) and different unconditioned stimuli (USs) (water, sucrose and sodium chloride) can be easily performed in a very similar experimental setting, which greatly facilitates studies on stimulus parameters to achieve various forms of conditioning and its underlying neurotransmitter mechanisms.

\section{Experimental procedures to study learning and memory in crickets}

We developed two procedures for the study of classical conditioning in crickets. One procedure is a "classical conditioning and operant testing procedure", which is based on the transfer of memory formed during classical conditioning to an operant testing situation (Matsumoto \& Mizunami, 2002). For conditioning, crickets were individually placed in a beaker. A filter paper soaked with an odor (CS) was approached to the head of the cricket, and then a drop of water or $20 \%$ sodium chloride solution (appetitive or aversive US) was applied to the mouth (Fig. 1A). In the operant relative odor preference test, crickets were individually placed in a test chamber and allowed to freely visit two odor sources, one of which was the conditioned odor and the other was the control odor (Fig. 1B). The time that the cricket explored each odor source with its
A

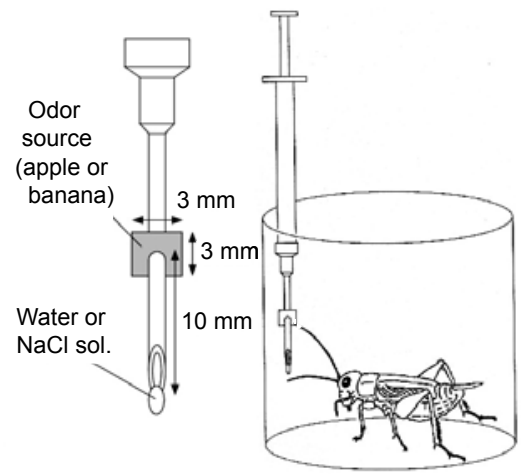

B

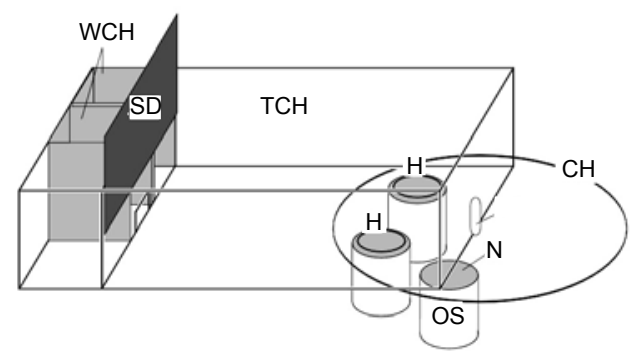

Figure 1. Procedures for olfactory conditioning in crickets. (A) For appetitive or aversive conditioning, an odor (e.g., apple or banana odor) was paired with water (appetitive US) or $20 \%$ sodium chloride solution (aversive US). A syringe containing water or sodium chloride solution was used for US delivery. A filter paper soaked with apple or banana essence was attached to the needle of the syringe. The filter paper was approached to the cricket's antennae, and then water or sodium chloride solution was presented to the mouth. (B) Apparatus for the odor preference test. Two holes $(\mathrm{H})$ connecting the chamber with odor sources (OS) were made on the floor of the test chamber (TCH). Each odor source consisted of a container with a filter paper soaked with apple or banana essence, covered with fine gauze net $(\mathrm{N})$. Three containers were mounted on a rotating container holder $(\mathrm{CH})$ and two of three odor sources could be presented at the same time. A cricket was placed in the waiting chamber (WCH) for 4 min for acclimation and then allowed to enter the test chamber to visit odor sources, by opening a sliding door (SD). Two minutes later, the relative positions of the apple and banana sources were changed. The preference test lasted for $4 \mathrm{~min}$. Modified from Matsumoto \& Mizunami (2002).

mouth or palpi was measured for evaluation of relative odor preference of the crickets. This procedure allows study of appetitive conditioning and aversive conditioning in a very similar experimental situation.

For visual pattern conditioning, either a white-center and black-surround pattern or a 
black-center and white-surround pattern was presented and then water or sodium chloride solution was presented to the mouth (Unoki, Matsumoto, \& Mizunami, 2006). In the visual pattern preference test, the two visual patterns were simultaneously presented on the wall of the test chamber, and the time that the cricket touched each of the patterns was recorded for evaluating relative preference between the two patterns.

The other procedure is conditioning of maxillary palpi extension response with an odor, which allowed evaluation of acquisition process (Matsumoto, Matsumoto, Wakuda, Ichihara, \& Mizunami, 2015). Crickets often extend their maxillary palpi and vigorously swing them when water is applied to the antennae, which we refer to as the maxillary palpi extension response (MER). Some odors such as vanilla and maple odors easily evoked MERs, while other odors such as peppermint and apple odors rarely induced MERs. We showed that the MER to an odor is increased by pairing of the odor with water US. This is analogous to olfactory conditioning of proboscis extension responses (PERs) in honey bees, in which pairing of an odor and sucrose US leads to an increase of the PER (Menzel \& Giurfa, 2006; Matsumoto, Sandoz, Devaud, Lormant, Mizunami, \& Giurfa, 2014). We also showed that the MER to an odor is decreased by pairing of the odor with sodium chloride US, thus allowing study of appetitive conditioning and aversive conditioning in a similar experimental situation.

\section{Roles of OA neurons and DA neurons in appetitive and aversive learning}

We investigated the effects of $\mathrm{OA}$ and DA receptor antagonists on appetitive and aversive olfactory conditioning in crickets (Unoki, Matsumoto, \& Mizunami, 2005). A previous study using honey bees (Hammer \& Menzel, 1998) and previous studies using the fruit-fly Drosophila melanogaster (Schwaerzel, Monastirioti, Scholz, Friggi-Grelin, Birman, \& Heisenberg, 2003) suggested that OA and DA neurons play roles in appetitive and aversive olfactory conditioning, respectively. Thus, we examined whether this is also the case in crickets. Crickets injected with an OA receptor antagonist (epinastine or mianserin) into the hemolymph prior to conditioning exhibited complete impairment of appetitive conditioning of an odor with water. On the other hand, these animals exhibited normal aversive conditioning with sodium chloride. This indicates that OA receptor antagonists do not impair sensory function, motor function or motivation necessary for learning. The results suggested that $\mathrm{OA}$ neurons are specifically involved in conveying water US. We also found that injection of a DA receptor antagonist (fluphenazine, chlorpromazine, spiperone or flupentixol) completely impaired aversive learning with sodium chloride US. In contrast, it did not impair appetitive learning with water. This indicates that DA receptor antagonists do not impair sensory function, motor function or motivation necessary for learning. These results suggest that DA neurons are specifically involved in conveying sodium chloride. We concluded that $\mathrm{OA}$ and DA neurons convey information about appetitive and aversive US, respectively, for olfactory conditioning in crickets.

We next studied the effects of $\mathrm{OA}$ and DA receptor antagonists on appetitive and aversive conditioning of a visual pattern (Unoki, Matsumoto, \& Mizunami, 2006) and a color cue (Nakatani, Matsumoto, Mori, Hirashima, Nishino, Arikawa, \& Mizunami, 2009). For conditioning of a visual pattern, crickets injected with an $\mathrm{OA}$ receptor antagonist into the hemolymph exhibited an impairment of appetitive learning, whereas aversive learning of a visual pattern was unaffected. In contrast, a DA receptor antagonist impaired aversive learning, but appetitive learning was unaffected. In color conditioning, injection of an OA receptor antagonist impaired appetitive color learning without affecting aversive color learning. In contrast, injection of a DA receptor antagonist impaired aversive color learning without affecting appetitive color learning. These results indicate that the roles 
of OA and DA neurons in conveying information about appetitive and aversive US, respectively, are ubiquitous in learning of odor, visual pattern and color stimuli. Thus, we concluded that $\mathrm{OA}$ and DA neurons mediate appetitive and aversive signals for various forms of learning in crickets. On the other hand, we noted that crickets are capable of associating different CSs and that this association occurs without participation of OA or DA neurons (Matsumoto, Hirashima, \& Mizunami, 2013).

Roles of $\mathrm{OA}$ neurons and DA neurons in mediating appetite and aversive reinforcement signals studied by using RNAi and transgenic crickets

Recent studies on the transgenic fruit-fly Drosophila melanogaster using sucrose and electric-shock US, however, have yielded a different picture (Liu, Plaçais, Yamagata, Pfeiffer, Aso, Friedrich, Siwanowicz, Rubin, Preat, \& Tanimoto, 2012; Burke, Huetteroth, Owald, Perisse, Krashes, Das, Gohl, Silies, Certel, \& Waddell, 2012). In the fruit-fly, different sets of DA neurons mediate both appetitive and aversive signals via the type 1 dopamine receptor Dop1. In this framework, OA neurons have only a peripheral role for sweet-taste sensing as their signals are relayed to DA neurons, which convey this information to the lobes of the mushroom bodies (MBs), in which olfactory CS and electric shock or sucrose US converge (Liu, Plaçais, Yamagata, et al., 2012; Burke, Huetteroth, Owald, et al., 2012; Waddell, 2013). Thus, a critical difference between flies and crickets is that DA neurons mediate appetitive signals in flies but not in crickets. We considered three possible reasons for this discrepancy, which we investigated in the cricket. The first possible reason is different methods used to knockdown DA signaling: while transgenesis provides a sophisticated way to silence neurotransmitter signaling in the fly, specificities of pharmacological antagonists used in the cricket are not perfect (Beggs, Tyndall, \& Mercer, 2011). The second possible reason is different kinds of appetitive US used. We used water in our studies on crickets, whereas sucrose was used in almost all studies on fruit-flies except for one using water (Lin, Owald, Chandra, Talbot, Huetteroth, \& Waddell, 2014). We thus paid attention to the possibility that DA conveys sucrose US but not water US in crickets. The third possible reason is that reinforcing mechanisms may not be uniform among insects. This possibility was thought to be less likely since it is generally believed that basic mechanisms of learning and memory are conserved among insect species.

In order to resolve the issue discussed above, we used the clustered regularly interspaced short palindromic repeats (CRISPR)/CRISPR-associated protein 9 (Cas9) system to produce crickets with knockout of the Dop1 gene (Awata, Watanabe, Hamanaka, et al., 2015). We found that Dop1 knockout crickets exhibited impairment in aversive learning with sodium chloride but exhibited no impairment in appetitive learning with water or sucrose. The latter finding indicates that the impairment was not due to impairment of sensory or motor functions or motivation necessary for learning and for responding to the conditioned odor in the post-training test. We thus conclude that Dop1 participates in aversive learning but not in appetitive learning in crickets. This differed from findings in fruit-flies that Dop1 is required for both appetitive and aversive learning (Liu, Plaçais, Yamagata, et al., 2012; Burke, Huetteroth, Owald, et al., 2012).

For further clarification of this issue, we investigated the effects of silencing of expression of genes that code the OA1 octopamine receptor and Dop1 and Dop2 dopamine receptors by RNAi in crickets (Awata, Wakuda, Ishimaru, et al., 2016). In this study, we used olfactory conditioning of maxillary palpi extension response (MER) to evaluate the effect of RNAi on acquisition process. Crickets were injected with dsRNA of $O A 1, D o p 1$ or Dop2 and two days later they were subjected to conditioning trials to associate an odor with water or sodium 
chloride. $O A 1$-silenced crickets exhibited complete impairment in appetitive learning with water, but they exhibited normal scores in aversive learning with sodium chloride. In contrast, Dop1-silenced crickets exhibited complete impairment in aversive learning but exhibited normal scores in appetitive learning. Dop2-silenced crickets showed normal scores in both appetitive learning and aversive learning. The results indicate that OA neurons mediate appetitive signals via OA1 receptors and that DA neurons mediate aversive signals via Dop1 receptors in crickets, providing decisive evidence that neurotransmitters and receptors that mediate appetitive signals indeed differ among different species of insects.

In mammals, there is evidence suggesting that midbrain DA neurons convey both appetitive and aversive signals in appetitive and aversive learning (Schultz, 2006). Thus, the roles of DA in mediating aversive signals are conserved among crickets, flies and mammals, whereas biogenic amines mediating appetitive signals are not the same among them.

\section{Participation of $\mathrm{OA}$ neurons and DA neurons in execution of appetitive and aversive conditioned responses}

We next studied the effects of $\mathrm{OA}$ and DA receptor antagonists on execution of conditioned responses (CRs) (or memory retrieval) after appetitive or aversive conditioning (Mizunami, Unoki, Mori, Hirashima, Hatano, \& Matsumoto, 2009). Crickets were subjected to appetitive or aversive olfactory conditioning and then they were injected with an OA or DA receptor antagonist before a retention test. Crickets injected with an OA receptor antagonist (epinastine) exhibited no $\mathrm{CR}$ to the appetitively conditioned odor, while they exhibited normal $\mathrm{CR}$ to the aversively conditioned odor. On the other hand, injection of a DA receptor antagonist (flupentixol) completely impaired execution of aversive $\mathrm{CR}$, but it had no effect on execution of appetitive CR. This is in accordance with the finding in honey bees that disruption of OA-ergic transmission in the antennal lobe, the primary olfactory center, by an OA receptor antagonist (mianserin) or by RNAi of the OA1 gene disrupted execution of appetitive $\mathrm{CR}$ (or appetitive memory retrieval) (Farooqui, Robinson, Vaessin, \& Smith, 2003). Moreover, injection of an $\mathrm{OA}$ receptor antagonist and injection of a DA receptor antagonist impaired execution of appetitive and aversive CRs, respectively, in visual pattern conditioning (Mizunami, Unoki, Mori, et al., 2009). Therefore, we concluded that OA neurons play a critical role in execution of appetitive CRs and that DA neurons play a critical role in execution of aversive CRs after conditioning of olfactory and visual signals.

Our findings are not consistent with conventional neural models of insect classical conditioning proposed in a study of the fruitfly. Figure 2A depicts a model proposed by Schwaerzel, Monastirioti, Scholz, et al., (2003) to account for the roles of intrinsic neurons (Kenyon cells) and extrinsic neurons of the mushroom body (MB) in conditioning of an odor with sucrose or electric shock in the fly. In this model, it is assumed that (1) "CS" neurons (Kenyon cells) that convey signals about a CS make synaptic connections with dendrites of "CR" neurons (output neurons of the MB lobe), activation of which leads to a CR, but these synaptic connections are silent or very weak before conditioning, (2) $\mathrm{OA}$-and DA-ergic neurons projecting to the lobes ("OA/DA" neurons), which convey signals for appetitive and aversive US, respectively, make synaptic connections with axon terminals of "CS" neurons, and (3) the efficacy of synaptic transmission from "CS" neurons to "CR" neurons that induces a $\mathrm{CR}$ (CS-CR connection) is strengthened by coincident activation of "CS" neurons and "OA/ DA" neurons during conditioning.

We have proposed a new model (Mizunami, Unoki, Mori, et al., 2009), with minimal modifications of the model proposed for the fruit-fly. In our model (Fig. 2B), we assumed that (1) activation of "OA/DA" neurons and resulting release of $\mathrm{OA}$ or $\mathrm{DA}$ are needed to 
A

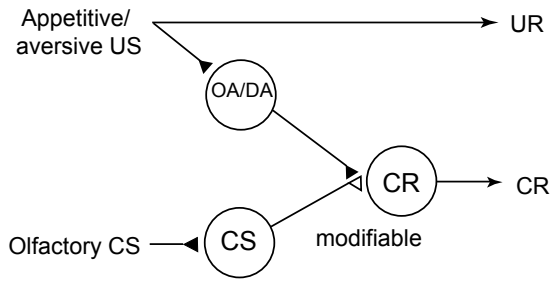

B

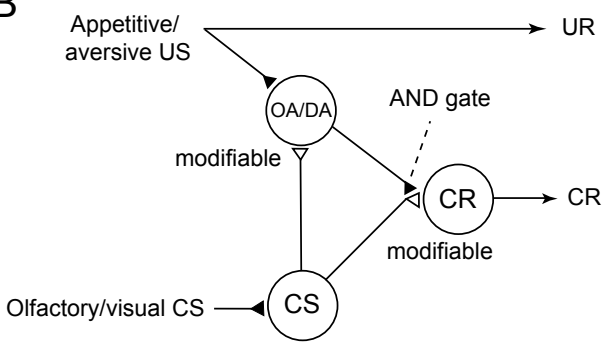

Figure 2. Conventional and new models of classical conditioning in insects. (A) A model proposed to account for the roles of intrinsic and extrinsic neurons of the mushroom body in olfactory conditioning in fruit-flies (Schwaerzel, et al., 2003). OA and DA neurons ("OA/DA" neurons) convey signals for appetitive and aversive US, respectively. "CS" neurons, which convey signals for CS, make synaptic connections with "CR" neurons that induce a CR, which mimics an unconditioned response (UR), the efficacy of the synaptic connection being strengthened by conditioning. "OA/DA" neurons make synaptic connections with axon terminals of "CS" neurons. (B) Our model of classical conditioning in crickets (Mizunami, et al., 2009). The model assumes that efficacy of synaptic transmission from "CS" neurons to "OA/DA" neurons is strengthened by conditioning and that coincident activation of "OA/DA" neurons and "CS" neurons is needed to activate "CR" neurons to lead to a CR (AND gate). Synapses the efficacies of which are modifiable by CS-US pairings are shown as open triangles. Modified from Mizunami, Unoki, Mori, et al. (2009).

"gate" the synaptic pathway from "CS" neurons to "CR" neurons after conditioning and (2) the synaptic connection from "CS" neurons to "OA/DA" neurons representing US is strengthened by coincident activation of "CS" neurons and "OA/DA" neurons by pairing of a CS with a US. The results of our pharmacological analysis using a secondorder conditioning procedure fully supported this model (Mizunami, Unoki, Mori, et al., 2009).

\section{Roles of OA neurons in mediating reward prediction error signals}

Understanding computational rules underlying associative learning is a major goal of neuroscience. In associative learning in mammals, it is widely accepted that the discrepancy, or error, between the actual US and the predicted US determines whether learning occurs when a stimulus is paired with the US (Schultz, 2006). This theory stems from the finding of "blocking" by Kamin (1969). He observed, in rats, pairing of stimulus A with US and subsequent pairing of stimuli A and B (another stimulus) with the US blocked association of stimulus B with the US. Kamin (1969) argued that the blocking is due to the requirement of surprise for learning, i.e., no learning occurs when the US is fully predicted. Subsequently, Rescorla and Wager (1972) formulated this proposition into the prediction error theory. Recent neuroscience research in mammals has demonstrated that activities of DA neurons in the ventral tegmental area of the midbrain mediate prediction error signals in classical conditioning (Schultz, 2006) and instrumental conditioning (Waelti, Dickinson, \& Schultz, 2001)

Unambiguous demonstration of the prediction error theory, however, has not been achieved in any learning systems. Blocking can also be accounted for by theories other than the prediction error theory (Miller, Barnet, \& Grahame, 1995; Pearce, 2008), and experiments are therefore needed to discriminate among different theories. The most influential theory is the attentional theory (or theory of attention) proposed by Mackintosh (1975) and Pearce \& Hall (1980), which accounts for blocking by a loss of attention to a stimulus. Another notable theory is the comparator hypothesis (Miller \& Matzel, 1988), which accounts for blocking by cue competition during memory retrieval. Experiments have been performed to discriminate the prediction error theory from other theories in some learning systems (Miller, Barnet, \& Grahame, 1995; Pearce, 2008), but unequivocal evidence to reject all alternative 
theories has not been obtained in any learning systems.

We obtained unequivocal evidence of blocking in classical conditioning in crickets, for the first time in any insects (Terao, Matsumoto, \& Mizunami, 2015). Efforts have been directed to obtain evidence of blocking in honey bees, but it has been concluded that blocking is not a robust phenomenon in honey bees (Guerrieri, Lachnit, Gerber, \& Giurfa, 2005; Blaser, Couvillon, \& Bitterman, 2006, 2008). We observed no learning of a stimulus (B) by pairing of a compound of $B$ and another stimulus (A) with appetitive US (or reward $)(\mathrm{AB}+$ training) when it was preceded by $\mathrm{A}+$ training. We then performed experiments to discriminate the prediction error theory and the attentional theory. The results of our experiment with 1-trial $\mathrm{AB}+$ conditioning supported the prediction error theory but not the attentional theory.

In order to obtain further evidence for the prediction error theory, we constructed a neural circuitry model of classical conditioning that is consistent with the prediction error theory (Fig. 3A), by revising our previous model (Fig. 2B). How this model accounts for blocking is illustrated in Fig. 3B. The model predicts that application of an $\mathrm{OA}$ receptor antagonist before $\mathrm{B}+$ training impairs learning of $\mathrm{B}$ but not formation of reward prediction by $B$ (see legend of Fig. 3). In accordance with this prediction, no learning of $\mathrm{B}$ occurred with subsequent $\mathrm{B}+$ training. The finding of "auto-blocking" in crickets can be accounted for by the prediction error theory but not by any other competitive theories to account for blocking, providing rigorous evidence for validity of the prediction error theory. Moreover, the results suggest that $\mathrm{OA}$ neurons mediate reward prediction error signals in crickets. Neural circuit mechanisms for computation of the prediction error remain unknown in any learning systems, and crickets should emerge as pertinent models in which to elucidate this important subject.

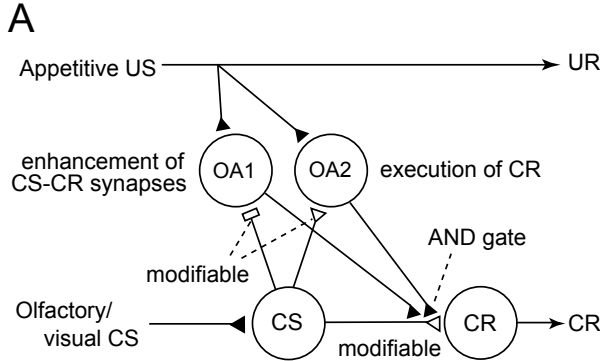

B

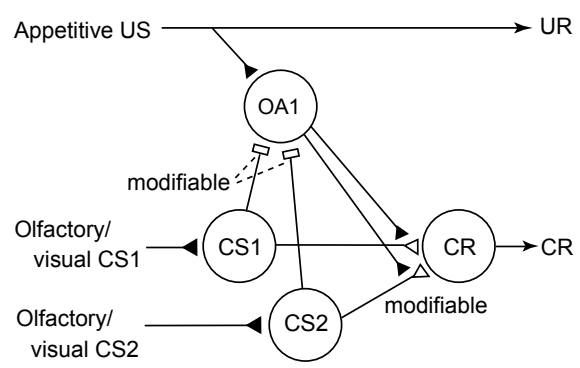

Figure 3. Our new model of the roles of OA neurons in appetitive conditioning to match the prediction error theory (Terao, et al., 2015), established by modifying our previous model (Mizunami, et al., 2009). We assumed the presence of "OA1" neurons that govern enhancement of "CS-CR" synapses (but not execution of $\mathrm{CR}$ ) in addition to "OA2" neurons that govern execution of $\mathrm{CR}$ or memory retrieval. OA2 neurons, but not OA1 neurons, govern the "AND gate". "OA1" neurons are assumed to receive silent or very weak inhibitory synaptic input from "CS" neurons before training, which are strengthened by CS-US pairing. During training, "OA1" neurons receive excitatory synaptic input (triangles) representing actual US and inhibitory input (rectangles) from "CS" neurons representing US predicted by CS, and thus their activities represent US prediction errors, thereby allowing US prediction error signals to govern enhancement of synaptic transmission. (B) Accounts for blocking by our model. The model assumes that pairing of a stimulus (CS1) with appetitive US leads to (1) an enhancement of inhibitory pathways from "CS1" neurons to "OA1" neurons and (2) that of excitatory synapses from "CS1" neurons to "CR" neurons. During pairing of a compound of CS1 and CS2 with US after sufficient repetition of CS1-US pairing, "OA1" neurons are inhibited by activation of "CS1" neurons and thus responses of "OA1" neurons to US are diminished. As a result, no enhancement of "CS2-OA1" synapses and "CS2-CR"synapses occurs, in which "CS2" are neurons mediating CS2. Thus, no learning of CS2 occurs. "OA2" neurons are not illustrated in this figure for simplicity. Synapses the efficacies of which are modifiable by CS-US pairings are shown as open rectangles or triangles. Modified from Terao, Matsumoto, \& Mizunami (2015). 


\section{Conclusions}

We conclude, at first, that neurotransmitter mechanisms of classical conditioning are, in part, conserved between insects and mammals. DA mediates aversive reinforcement in crickets, flies and mammals, whereas neurotransmitters mediating appetitive signals are not the same among different animals. More studies on the roles of aminergic neurons in learning in various species of vertebrates and invertebrates are needed to elucidate the diversity and evolution of basic neurotransmitter mechanisms of associative learning. Secondly, we showed that basic computational rules underlying classical conditioning are greatly conserved among insects and mammals. Associative learning is governed by prediction error signals in both insects and mammals. More studies to anatomically and physiologically characterize $\mathrm{OA}$ and DA neurons involved in learning in crickets are needed to elucidate basic neural circuit mechanisms of prediction error computation.

\section{Acknowledgements}

This study was supported by Grants-inAid for Scientific Research from the Ministry of Education, Science, Culture, Sports and Technology of Japan to M. M. (No.16H04814 and 16K18586).

\section{References}

Awata, H., Wakuda, R., Ishimaru, Y., Matsuoka, Y., Terao, K., Katata, S., Matsumoto, Y., Hamanaka, Y., Noji, S., Mito, T., \& Mizunami, M. 2016 Roles of OA1 octopamine receptor and Dop1 dopamine receptor in mediating appetitive and aversive reinforcement revealed by RNAi studies. Scientific Reports, 6, 29696. Awata, H., Watanabe, T., Hamanaka, Y., Mito, T., Noji, S., \& Mizunami, M. 2015 Knockout crickets for the study of learning and memory: Dopamine receptor Dop1 mediates aversive but not appeti- tive reinforcement in crickets. Scientific Reports, 5, 15885.

Beggs, K. T., Tyndall, J. D., \& Mercer, A. R. 2011 Honey bee dopamine and octopamine receptors linked to intracellular calcium signaling have a close phylogenetic and pharmacological relationship. PLoS One, 6, e26809.

Blaser, R. E., Couvillon, P. A., \& Bitterman, M. E. 2006 Blocking and pseudoblocking: new control experiments with honeybees. Quarterly Journal of Experimental Psychol ogy, 59, 68-76.

Blaser, R. E., Couvillon, P. A., \& Bitterman, M. E. 2008 Within-subjects experiments on blocking and facilitation in honeybees (Apis mellifera). Journal of Comparative Psychology, 122, 373-378.

Burke, C. J., Huetteroth, W., Owald, D., Perisse, E., Krashes, M. J., Das, G., Gohl, D., Silies, M., Certel, S., \& Waddell, S. 2012 Layered reward signalling through octopamine and dopamine in Drosophila. Nature, 492, 433-437.

Farooqui, T., Robinson, K., Vaessin, H., \& Smith, B. H. 2003 Modulation of early olfactory processing by an octopaminergic reinforcement pathway in the honeybee. Journal of Neuroscience, 23, 5370-5380.

Guerrieri, F., Lachnit, H., Gerber, B., \& Giurfa, M. 2005 Olfactory blocking and odorant similarity in the honeybee. Learning \& Memory, 12, 86-95.

Hammer, M. R., \& Menzel, R. 1998 Multiple sites of associative odor learning as revealed by local brain microinjections of octopamine in honeybees. Learning \& Memory, 5, 146-156.

Kamin, L. 1969 Predictability, surprise, attention and conditioning. In B. A. Campbell \& R. M. Church (Eds.), Punishment and aversive behavior. New York: AppletonCentury-Crofts. Pp.279-298.

Lin, S., Owald, D., Chandra, V., Talbot, C., Huetteroth, W., \& Waddell, S. 2014 Neural correlates of water reward in thirsty Drosophila. Nature Neuroscience, 17, 1536-1542.

Liu, C., Plaçais, P. Y., Yamagata, N., Pfeiffer, B. 
D., Aso, Y., Friedrich, A. B., Siwanowicz, I., Rubin, G. M., Preat, T., \& Tanimoto, H. 2012 A subset of dopamine neurons signals reward for odour memory in Drosophila. Nature, 488, 512-516.

Mackintosh, N. J. 1975 A theory of attention: Variations in the associability of stimuli with reinforcement. Psychological Reviews, 82, 276-298.

Matsumoto, Y., Hatano, A., Unoki, S., \& Mizunami, M. 2009 Stimulation of the cAMP system by the nitric oxide-cGMP system underlying the formation of longterm memory in an insect. Neuroscience Letters, 467, 81-85.

Matsumoto, Y., Hirashima, D., \& Mizunami, M. 2013 Analysis and modeling of neural processes underlying sensory preconditioning. Neurobiology of Learning and Memory, 101, 103-113.

Matsumoto, Y., Matsumoto, C. S., Wakuda, R., Ichihara, S., \& Mizunami, M. 2015 Roles of octopamine and dopamine in appetitive and aversive memory acquisition studied in olfactory conditioning of maxillary palpi extension response in crickets. Frontiers in Behavioral Neuroscience, 9, 230.

Matsumoto, Y., \& Mizunami, M. 2002 Temporal determinants of olfactory long-term retention in the cricket Gryllus bimaculatus. Journal of Experimental Biology, 205, 1429-1437.

Matsumoto, Y., Sandoz, J. C., Devaud, J. M., Lormant, F., Mizunami, M., \& Giurfa, M. 2014 Cyclic nucleotide - gated channels, calmodulin, adenylyl cyclase and calcium/ calmodulin-dependent kinase II are required for late but not early long-term memory formation in the honey bee. Learning \& Memory, 21, 272-286.

Matsumoto, Y., Unoki, S., Aonuma, H., \& Mizunami, M. 2006 Critical roles of the nitric oxide-cGMP cascade in the formation of cAMP-dependent long-term memory. Learning \& Memory, 13, 35-44.

Menzel, R., \& Giurfa, M. 2006 Dimensions of cognition in an insect, the honeybee. Behavioral and Cognitive Neuroscience
Reviews, 5, 24-40.

Miller, R. R., Barnet, R. C., \& Grahame, N. J. 1995 Assessment of the Rescorla-Wagner model. Psychological Bulletin, 117, 363-386.

Miller, R. R., \& Matzel, L. D. 1988 The comparator hypothesis: a response rule for the expression of associations. Psychology of Learning and Motivation, 22, 51-92.

Mizunami, M., \& Matsumoto, Y. 2010 Roles of aminergic neurons in formation and recall of associative memory in crickets. Frontiers in Behavioral Neuroscience, 4, 172.

Mizunami, M., Matsumoto, Y., Watanabe, H., \& Nishino, H. 2013 Chapter 41: Olfactory and visual learning in cockroaches and crickets. In R. Menzel \& P. R. Benjamin (Eds.), Invertebrate Learning and Memory, Berlin: Springer. Pp.547-558.

Mizunami, M., Unoki, S., Mori, Y., Hirashima, D., Hatano, A., \& Matsumoto, Y. 2009 Roles of octopaminergic and dopaminergic neurons in appetitive and aversive memory recall in an insect. BMC Biology, 7, 46.

Mizunami, M., Yokohari, F., \& Takahata, M. 1999 Exploration into the adaptive design of the arthropod "microbrain." Zoological Science, 16, 703-709.

Mizunami, M., Yokohari, F., \& Takahata, M. 2004 Further exploration into the adaptive design of the arthropod "microbrain": I. Sensory and memory-processing systems. Zoological Science, 21, 1141-1151.

Nakatani, Y., Matsumoto, Y., Mori, Y., Hirashima, D., Nishino, H., Arikawa, K., \& Mizunami, M. 2009 Why the carrot is more effective than the stick: Different dynamics of punishment memory and reward memory and its possible biological basis. Neurobiology of Learning and Memory, 92, 370-380.

Pearce, J. M. 2008 Associative learning. In Animal Learning \& Cognition. New York: Psychology press. Pp.35-91.

Pearce, J. M., \& Hall, G. 1980 A model for Pavlovian learning: Variations in the effectiveness of conditioned but not of 
unconditioned stimuli. Psychological Review, 87, 532-552.

Rescorla, R. A., \& Wagner, A. R. 1972 A theory of Pavlovian conditioning: Variations in the effectiveness of reinforcement and nonreinforcement. In A. Black \& W. R. Prokasy (Eds.), Classical Conditioning II. New York: Academic Press. Pp.64-99.

Schultz, W. 2006 Behavioral theories and the neurophysiology of reward. Annual Reviews in Psychology, 57, 87-115.

Schwaerzel, M., Monastirioti, M., Scholz, H., Friggi-Grelin, F., Birman, S., \& Heisenberg, M. 2003 Dopamine and octopamine differentiate between aversive and appetitive olfactory memories in Drosophila. Journal of Neuroscience, 23, 10495-10502.

Takahashi, T., Hamada, A., Miyawaki, K., Matsumoto, Y., Mito, T., Noji, S., \& Mizunami, M. 2009 Systemic RNA interference for the study of learning and memory in an insect. Journal of Neuroscience Methods, 179, 9-15.

Terao, K., Matsumoto, Y., \& Mizunami, M.
2015 Critical evidence for the prediction error theory in associative learning. Scientific Reports, 5, 8929.

Unoki, S., Matsumoto, Y., \& Mizunami, M. 2005 Participation of octopaminergic reward system and dopaminergic punishment system in insect olfactory learning revealed by pharmacological study. European Journal of Neuroscience, 22, 14091416.

Unoki, S., Matsumoto, Y., \& Mizunami, M. 2006 Roles of octopaminergic and dopaminergic neurons in mediating reward and punishment signals in insect visual learning. European Journal of Neuroscience, 24, 2031-2038.

Waddell, S. 2013 Reinforcement signalling in Drosophila; dopamine does it all after all. Current Opinion in Neurobiology, 23, 324329.

Waelti, P., Dickinson, A., \& Schultz, W. 2001 Dopamine responses comply with basic assumptions of formal learning theory. Nature, 412, 43-48.

(2017. 3.1 受稿, 2017. 5.11 受理) 